\title{
Helping patients and the emergency department: enhanced patient care and reduced hospital admission rates with a pilot rapid access neurology clinic (RANC)
}

\author{
Authors: Thomas Peukert, ${ }^{\mathrm{A}}$ Stella Hughes, ${ }^{\mathrm{A}}$ Richard Wilson, ${ }^{\mathrm{B}}$ Stephen Hunt, ${ }^{\mathrm{A}}$ Gavin McDonnell ${ }^{\mathrm{A}}$ \\ and John Craig ${ }^{\mathrm{A}}$
}

\section{Aims}

To assess the efficacy and acceptability of a rapid access neurology clinic (RANC) in improving access to neurological diagnosis and reducing hospital admissions and investigations for patients with neurological complaints presenting to the emergency department (ED).

\section{Methods}

A pilot RANC was commenced for 4 weeks in October 2013, to supplement a UK tertiary-level neurology service. Three clinics were held per week, with slots available for four patients per clinic. Appointments were filled directly from senior ED staff. Only patients that otherwise would have been admitted to the medical admissions unit (MAU) were eligible for referral to the RANC.

\section{Results}

A total of 45 patients were seen in the RANC, 28 of whom avoided admission owing to accessibility of an urgent neurology clinic. The average waiting time to be seen was 8 days. The majority of referrals to the RANC were due to headache or seizures. The number of CT or MRI scans arranged in the RANC was significantly lower than that for patients who were admitted to MAU ( 0.38 vs 1.15 scans per patient, respectively). Two-thirds of patients seen in the RANC did not require follow-up under neurology.

Questionnaires were sent out to patients that attended the RANC and to ED doctors. The response showed an extremely high satisfaction rate in both groups. The estimated cost savings by avoiding admissions were calculated as $\mathfrak{£} 46,200$ during the 4 weeks. This did not include the cost savings due to reduction in performing MRI/CT scans. Aside from cost savings, the overall quality of care for patients with neurological complaints is enhanced through rapid access to specialist services, expert opinions and timely diagnoses.
Authors: ${ }^{A}$ Department of Neurology, Royal Victoria Hospital, Belfast HSC Trust, Belfast, UK; ${ }^{B}$ Department of Emergency Medicine, Royal Victoria Hospital, Belfast HSC Trust, Belfast, UK

\section{Conclusions}

The results of the pilot study suggest that provision of a RANC can reduce unnecessary hospital admissions and investigations for patients with neurological complaints. The pilot study received very positive feedback from ED colleagues and patients. Regular provision of the service may lead to considerable cost savings.

\section{Conflict of interest statement}

The authors have no conflicts of interest relating to this project. 